\title{
Orbital and Pulmonary Actinomycosis: The First Case Report and Literature Review
}

\author{
Paruspak Payoong (iD, Natcha Saetiew, Opass Putcharoen, and Chusana Suankratay if \\ Division of Infectious Diseases, Department of Medicine, Faculty of Medicine, Chulalongkorn University, Bangkok, Thailand \\ Correspondence should be addressed to Chusana Suankratay; csuankratay@gmail.com
}

Received 15 May 2018; Accepted 9 July 2018; Published 26 July 2018

Academic Editor: Paul Horrocks

Copyright (c) 2018 Paruspak Payoong et al. This is an open access article distributed under the Creative Commons Attribution License, which permits unrestricted use, distribution, and reproduction in any medium, provided the original work is properly cited.

Orbital actinomycosis is a very rare clinical manifestation of orbital infection caused by Actinomyces species, anaerobic Grampositive filamentous bacteria. We report herein a case of a 58-year-old man who presented with chronic progressive course of total ophthalmoparesis in association with productive cough, leading to the diagnosis after extensive investigation. In addition, all reported cases of orbital actinomycosis in the literature are reviewed.

\section{Introduction}

Actinomyces species are anaerobic Gram-positive filamentous bacteria and ubiquitous in soil and microbiota of both animals and humans. The infection caused by Actinomyces species, actinomycosis, is usually indolent and slowly progressive [1]. There are wide clinical manifestations of actinomycosis depending on the infection site including orocervicofacial, thoracic, abdominal, and pelvic diseases [1]. To date, there have been handful cases of orbital actinomycosis. The source of infection is usually following the head injury or from the infection of adjacent paraorbital organs including paranasal sinus, nose, cavernous sinus, and brain. We report herein a case of a 58-year-old man who presented with chronic progressive course of total ophthalmoparesis in association with productive cough, leading to the diagnosis after extensive investigation.

\section{Case Report}

A 58-year-old Thai previously healthy male farmer, living in Roi Et Province (Northeast Thailand), was referred to our hospital, King Chulalongkorn Memorial Hospital, due to problems with total ophthalmoparesis and productive cough for 2 months. Two months prior to admission (PTA), he developed productive cough and low-grade fever with no response to many courses of antibiotic treatment. One month PTA, he noted binocular horizontal diplopia of the right eye, before progression to visual loss and ptosis despite antibiotic and steroid treatment. His past medical history was unremarkable except heavy alcoholic drinking.

The right eye examination revealed eyelid swelling, proptosis, marked chemosis, and complete ptosis; light perception of visual acuity; absent afferent pupillary reflex; and optic disk swelling and retinal hemorrhage. Neurological examination revealed right eye total ophthalmoparesis and decreased pinprick sensation of right cranial VI nerve area. Multiple dental caries were noted. Pulmonary examination revealed fine crackles at both the lungs. Other examination was unremarkable.

Complete blood count showed hemoglobin of $9.3 \mathrm{~g} / \mathrm{dL}$, white blood cells of 25,540 cells $/ \mathrm{mm}^{3}$ (85.1\% neutrophil, $9.8 \%$ lymphocyte, and $4.8 \%$ monocyte), and platelets of 697,000 cells $/ \mathrm{mm}^{3}$. Blood chemistry was normal. Anti-HIV test was negative. Chest $\mathrm{X}$-ray revealed bilateral diffuse reticulonodular and alveolar infiltrations. Orbital computed tomography revealed a $1.5 \times 2.9 \times 1.5 \mathrm{~cm}$ rim-enhancing hypodense lesion at the right orbital apex abutting to the optic nerve sheath complex and right medial rectus muscle (Figure 1).

A diagnosis of orbital and pulmonary infections was made, and emergent anterior orbitotomy was performed and 


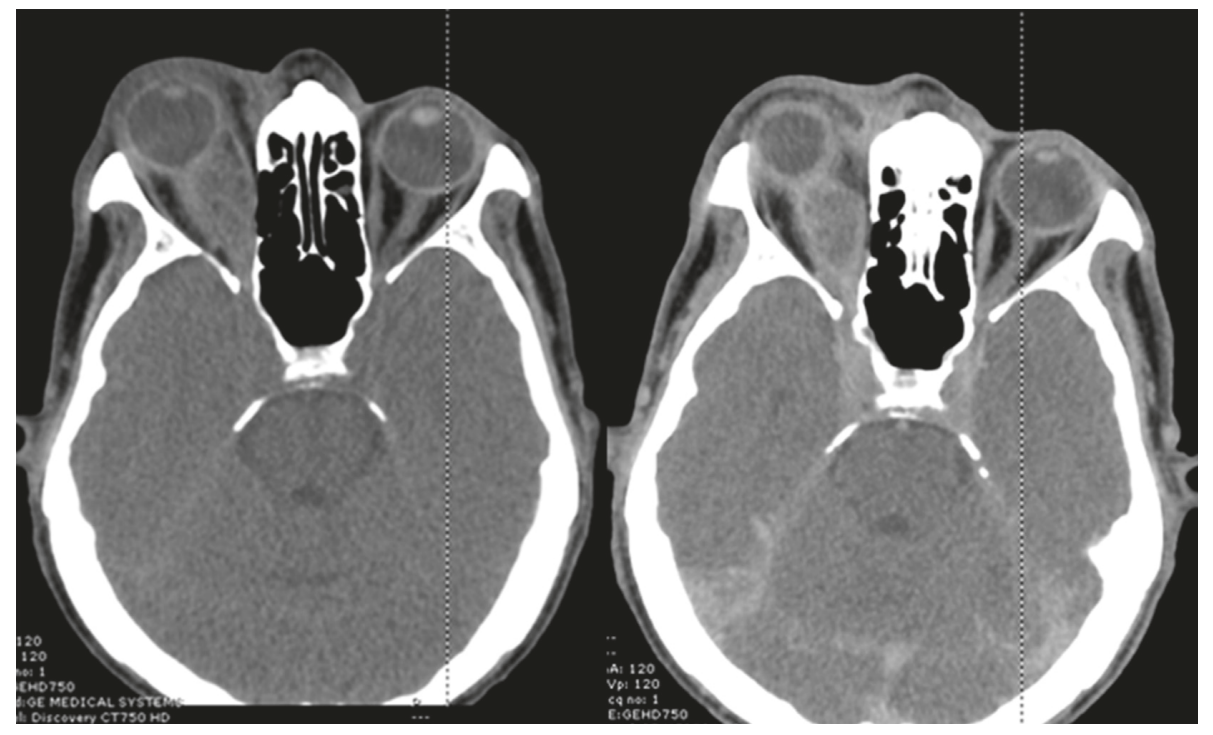

Figure 1: Computed tomography showing rim-enhancing hypodense lesion at the right orbital apex abutting to the optic nerve sheath complex and right medial rectus muscle.

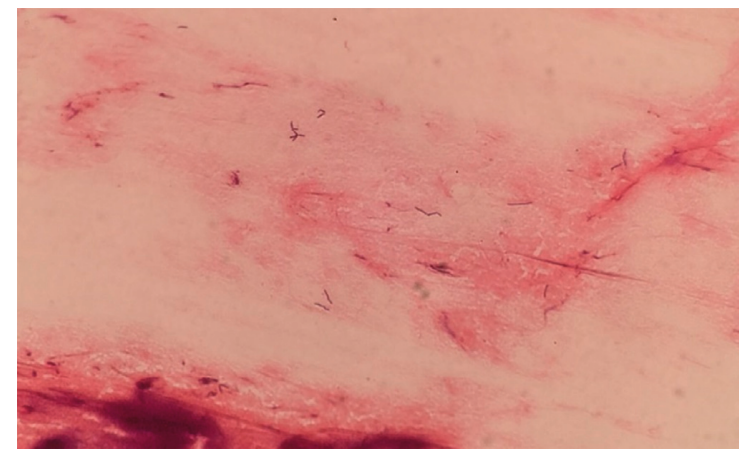

(a)

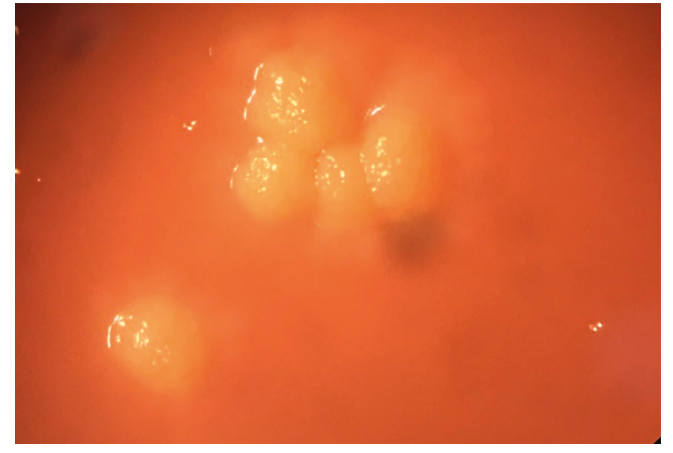

(b)

Figure 2: (a) The pus Gram stain exhibiting Gram-positive filamentous bacilli. (b) Molar tooth-like colonies of Actinomyces israelii.

revealed purulent discharge from the orbit. The pus and sputum Gram stain exhibited many Gram-positive filamentous bacilli, but non-acid-fast, on both standard and modified acid-fast bacilli stains. Hence, Actinomyces species was suspected, and Nocardia species could be excluded due to negative results on modified AFB stain. The pus cultures finally grew Actinomyces israelii (Figures 2(a) and 2(b)). Further bacterial identification by base sequencing of $16 \mathrm{~S}$ ribosomal DNA was A. israelii with 99\% identity (NCBI BLAST Search).

$\mathrm{He}$ was given penicillin $\mathrm{G}$ sodium of 24 million units per day for 6 weeks and amoxicillin of $2 \mathrm{~g}$ per day for another 12 months. After 10 days of treatment, there was a much improvement of lung infiltrates on his chest X-ray. The patient was doing well when last seen 1 year after discharge with mild residual impaired ophthalmoparesis and ptosis but still moderate visual impairment.

\section{Discussion}

To our knowledge, our case is the first report of both orbital and pulmonary actinomycosis. All previously reported cases were only orbital actinomycosis.
A portal of entry to the orbit in our case is probably from the hematogenous spread from the lungs, even though only unilateral orbit is involved. Due to sequence of respiratory and eye symptoms, the imaging reveals that there is no infection of paraorbital organs and presumed aspiration pneumonia from heavy alcohol drinking. In contrast, orbital actinomycosis in all previously reported cases was caused by infection in paraorbital organs or following the injury.

A literature review of all 10 cases (including our case) of orbital actinomycosis is shown in Table 1 . The median age is 45 (IQR 37, 56) years with the male: female ratio of $2: 1$. Europe and Asia are the most common continents (4, 40\% each), followed by North America and South America (1, $10 \%$ each). Teeth extraction history and chronic steroid use are the most common predisposing factors (4, 40\% each), followed by sinusitis $(2,20 \%)$ and trauma $(1,10 \%)$. The median duration of illness is 21 (IQR 11, 51) days. Ophthalmoparesis and ptosis are the most common presentations $(7,70 \%$ each), followed by proptosis $(4,40 \%)$ and visual loss $(3,30 \%)$. Six $(60 \%)$ patients are afebrile. On imaging, there are 5 and 3 extraconal and intraconal lesions ( 1 infraorbital nerve and 1 unknown data). Of 5 extraconal 


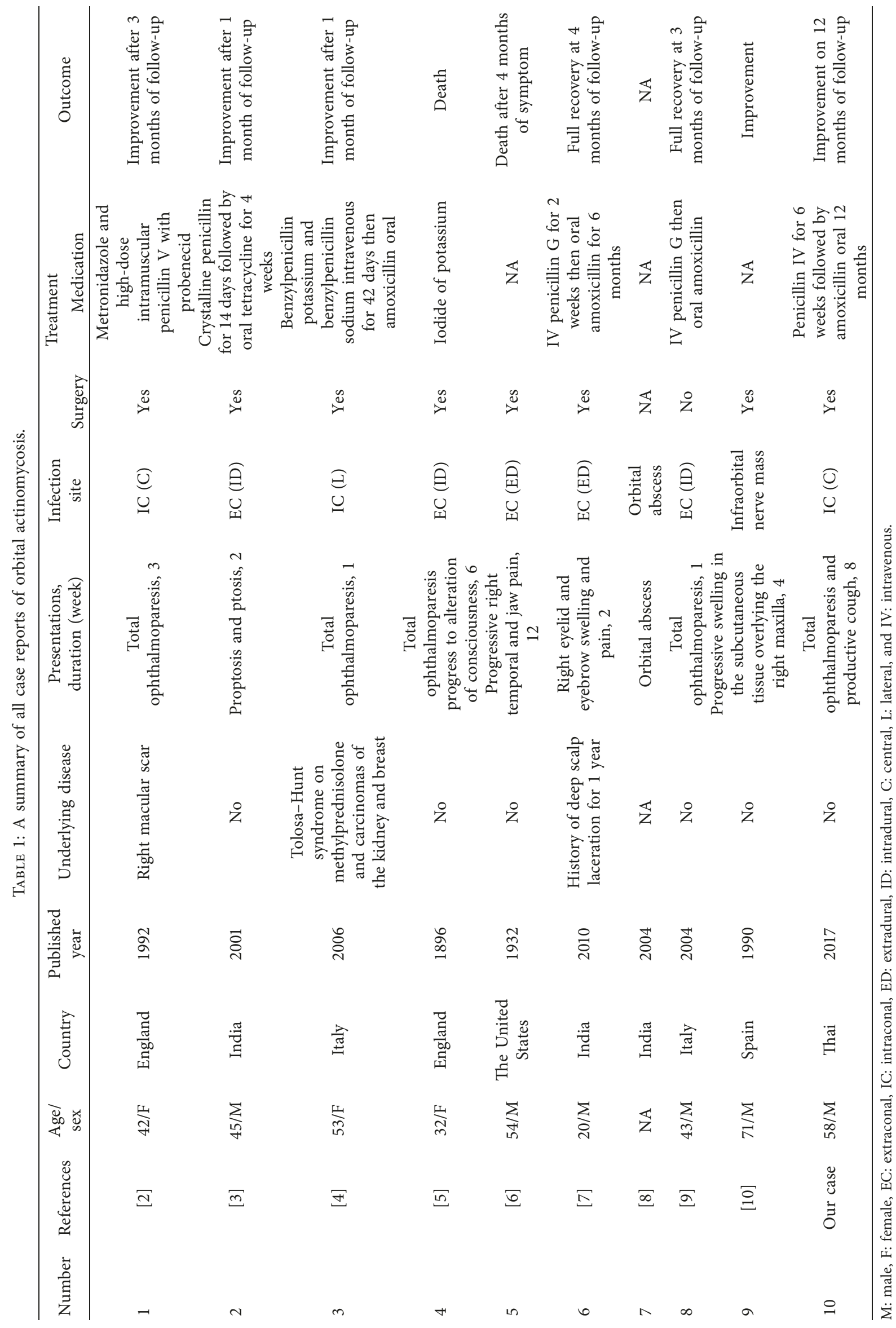


lesions, there are 3 and 2 intradural and extradural lesions. Of 3 intraconal lesions, there are 2 and 1 central and lateral lesions. Of 10 patients (no data in 1 patient), 8 (80\%) undergo surgical intervention. Penicillin is the most common prescribed antibiotic $(6,60 \%)$, followed by tetracycline and gentamicin (1, $10 \%$ each). There is $12 \%$ mortality rate. Full recovery and partial recovery are observed in $2(20 \%)$ and 6 (60\%) patients (no data in 2 patients).

\section{Conclusions}

To our knowledge, our case is the first report of both orbital and pulmonary actinomycosis, likely from hematogenous spread from the lungs.

\section{Conflicts of Interest}

The authors declare that there are no conflicts of interest regarding the publication of this paper.

\section{References}

[1] T. A. Russo, "Agents of actinomycosis," in Mandell, Douglas, and Bennett's Principles and Practice of Infectious Diseases, J. D. R. Bennett and M. Blaser, Eds., pp. 2863-2873, Elsevier Saunders, Toronto, ON, Canada, 2015.

[2] T. J. A. G. Sullivan and J. E. Wright, "Actinomycosis of the orbit," British Journal of Ophthalmology, vol. 76, no. 8, pp. 505-506, 1992.

[3] S. D. S. O. Nithyanandam, S. S. Rao, R. R. Battu, and S. George, "Rhinoorbitocerebral actinomycosis," Ophthalmic Plastic and Reconstructive Surgery, vol. 17, no. 2, pp. 134-136, 2001.

[4] L. C. L. Pagliani and G. M. Cavallini, "Orbital actinomycosis associated with painful ophthalmoplegia. Actinomycosis of the orbit," Ophthalmologica, vol. 220, no. 3, pp. 201-205, 2006.

[5] W. B. Ransom, "A case of actinomycosis of the orbit, with a summary of seven other cases of actinomycosis," BMJ, vol. 1, no. 1582, pp. 1553-1555, 1896.

[6] G. A. Leahey, "Orbital actinomycosis," Transactions of the American Ophthalmological Society, vol. 30, pp. 457-503, 1932.

[7] V. Hegde, N. Puthran, S. Mahesha, and B. Anupama, "A rare and an unusually delayed presentation of orbital actinomycosis following avulsion injury of the scalp," Indian Journal of Ophthalmology, vol. 58, no. 3, pp. 238-240, 2010.

[8] U. Dhaliwal, V. K. Arora, N. Singh, and A. Bhatia, "Clinical and cytopathologic correlation in chronic inflammations of the orbit and ocular adnexa: a review of 55 cases," Orbit, vol. 23, no. 4, pp. 219-225, 2004.

[9] J. Mandrioli, G. Frank, P. Sola et al., "Tolosa-hunt syndrome due to actinomycosis of the cavernous sinus: the infectious hypothesis revisited," Headache, vol. 44, no. 8, pp. 806-811, 2004.

[10] G. Har-El, D. A. Prager, F. De Soto La Paix, and M. Nash, "Actinomycotic granuloma masquerading as an infraorbital nerve neoplasm," Head \& Neck, vol. 12, no. 3, pp. 261-263, 1990. 


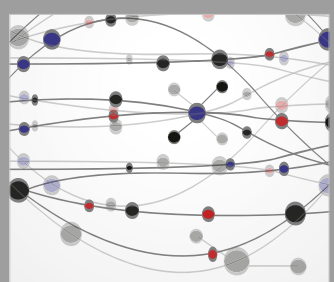

The Scientific World Journal
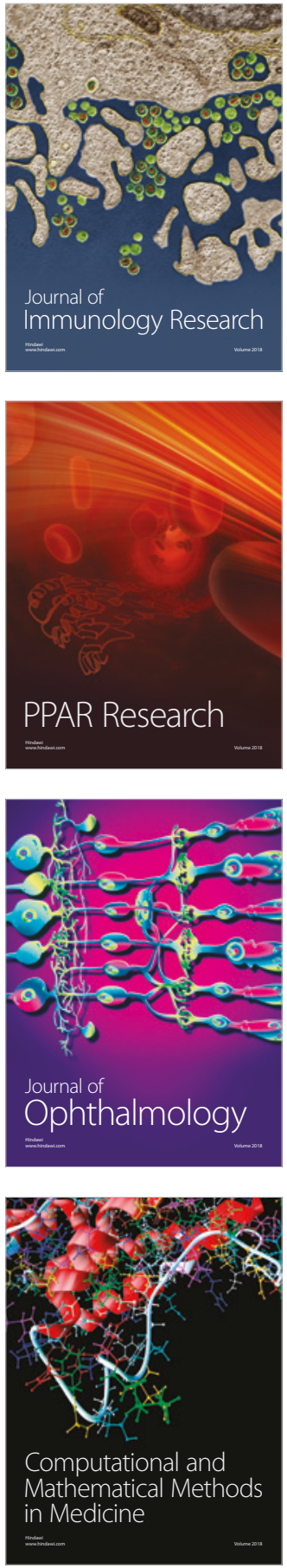

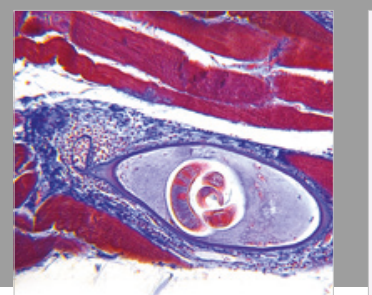

Gastroenterology Research and Practice

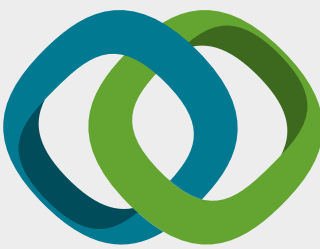

\section{Hindawi}

Submit your manuscripts at

www.hindawi.com
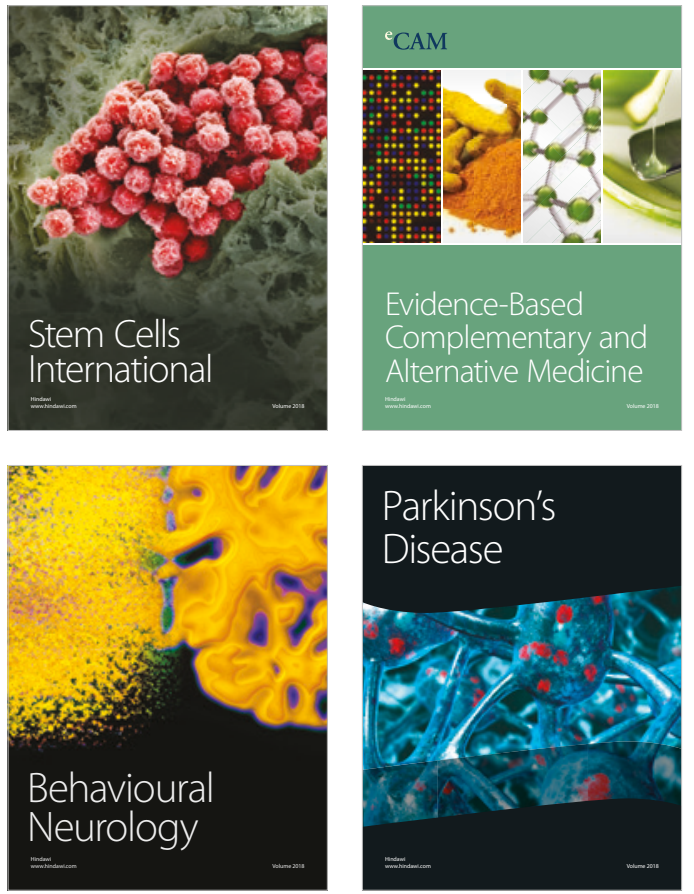

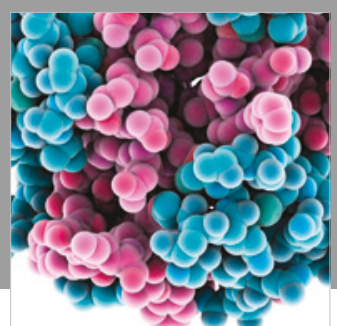

ournal of

Diabetes Research

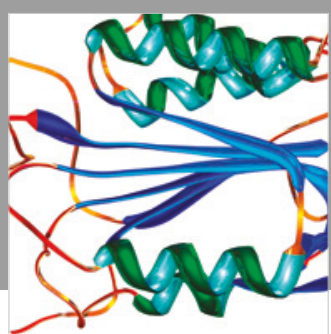

Disease Markers
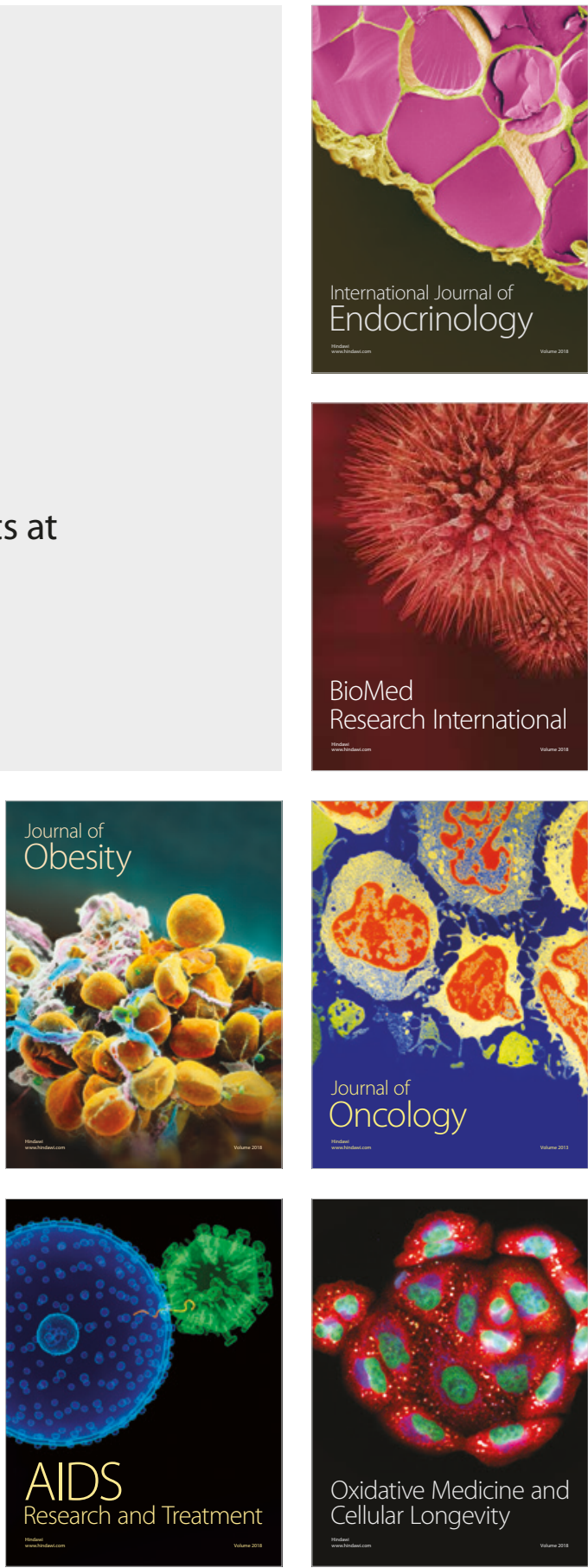